Société d'histoire de la révolution de 1848 et des révolutions du XIXe siècle

$52 \mid 2016$

Chrononymes. Dénommer le siècle

\title{
Une mobilisation internationale de masse à l'époque du Risorgimento : l'aide financière des catholiques français à la papauté (1860-1870)
}

An International Mass Mobilization at the Risogimiento period: the French Catholics' Financial Help to the Papacy (1860-1870)

Eine internationale Massenmobilisierung zur Zeit des Risorgimento: Die finanzielle Unterstützung französischer Katholiken für das Papsttum (1860-1870)

\section{Arthur Hérisson}

\section{CpenEdition}

\section{Journals}

Édition électronique

URL : http://journals.openedition.org/rh19/5005

DOI : $10.4000 /$ rh 19.5005

ISSN : $1777-5329$

Éditeur

La Société de 1848

Édition imprimée

Date de publication : 1 juin 2016

Pagination : 175-192

ISSN : 1265-1354

Référence électronique

Arthur Hérisson, « Une mobilisation internationale de masse à l'époque du Risorgimento : l'aide financière des catholiques français à la papauté (1860-1870) », Revue d'histoire du XIXe siècle [En ligne], 52 | 2016, mis en ligne le 01 juin 2019, consulté le 03 mai 2019. URL : http://journals.openedition.org/ rh19/5005; DOl : 10.4000/rh19.5005 


\section{ARTHUR HÉRISSON \\ Une mobilisation internationale de masse à l'époque du Risorgimento : l'aide financière des catholiques français à la papauté (1860-1870)}

Depuis plusieurs décennies, et singulièrement depuis les années 2000, de nombreux historiens se sont attachés à analyser le Risorgimento dans un cadre non plus simplement italien mais transnational ${ }^{1}$. En s'intéressant à des objets variés - de la diffusion des idées ${ }^{2}$ au volontariat militaire international ${ }^{3}-$, ils ont ainsi montré l'émergence d'un vaste espace politique transnational structuré par des circulations de types divers. Si celles des hommes et des idées ont largement retenu l'attention des chercheurs, il n'en est pas de même d'un autre type de circulation : les circulations d'argent.

Pourtant, chacun des camps qui s'affrontèrent dans la péninsule put compter sur des soutiens économiques importants venus de l'étranger. En France, des journaux favorables à l'unification comme Le Siècle et L'Opinion nationale lancèrent ainsi des souscriptions en faveur de Garibaldi ${ }^{4}$. La manifestation la plus importante d'une solidarité économique transnationale ne concerna cependant pas le camp des partisans de l'unité italienne. Durant toutes les années 1860 se produisit en effet une importante mobilisation des catholiques européens, qui offrit à l'État pontifical d'importants revenus.

1. Gilles Pécout, «Pour une lecture méditerranéenne et transnationale du Risorgimento ", Revue d'histoire du XIX'e siècle, $\mathrm{n}^{\circ}$ 44, janvier-juin 2012, p. 29-47.

2. Franco Venturi, "La circolazione delle idee e l'emigrazione", Rassegna storica del Risorgimento, tome $51, \mathrm{n}^{\circ} 2-3,1954$, p. 203-222. Sur la circulation des idées libérales : Maurizio Isabella, Risorgimento in Exile. Italian Emigres and the Liberal International in the Post-Napoleonic Era, Oxford, Oxford University Press, 2009 et Pierre-Marie Delpu, «Fraternités libérales et insurrections nationales : Naples et l'Espagne, 1820-1821", Revue d'histoire du XIXe siècle, $\mathrm{n}^{\circ}$ 49, juillet-décembre 2014, p. 195-213.

3. Chacun des camps s'affrontant dans la péninsule a pu compter sur le soutien de volontaires étrangers. Sur les volontaires français ayant combattu en faveur du Risorgimento en 1848-1849 : AnneClaire Ignace, Des quarante-huitards français en Italie. Étude sur la mobilisation de volontaires français pour le Risorgimento (1848-1849), thèse d'histoire sous la direction de Gilles Pécout et d'Alberto Mario Banti, Université Paris 1, 2010. Du côté des adversaires du Risorgimento, on pourra se reporter à Jean Guénel, La dernière guerre du pape. Les Zouaves pontificaux au secours du Saint-Siège (1860-1870), Rennes, Presses universitaires de Rennes, 1998 et à Simon Sarlin, Le légitimisme en armes. Histoire d'une mobilisation internationale contre l'unité italienne, Rome, École française de Rome, 2013. Le Journal of Modern Italian Studies a par ailleurs consacré en 2009 un numéro à la question de la place des volontaires internationaux dans le Risorgimento.

4. Ferdinand Boyer, "Souscriptions pour Garibaldi en France (1860)», Rassegna storica del Risorgmento, tome 47, $\mathrm{n}^{\circ} 1,1960$, p. 69-74. 
Cette mobilisation prit deux formes principales : l'envoi de dons destinés au pape ou à son armée et la souscription de titres des emprunts pontificaux. Grâce à cette aide, l'État pontifical, dont les revenus avaient fortement baissé après la perte des Romagnes en 1859 et des Marches et de l'Ombrie en 1860, réussit pendant dix ans à surmonter les graves problèmes financiers auxquels il était confronté.

L'histoire de cette mobilisation reste encore peu connue. Certes, Carlo Crocella l'a étudiée à l'échelle européenne ${ }^{5}$ tandis qu'existent quelques monographies qui retracent à l'échelle d'un diocèse ou d'une région les étapes de la collecte ${ }^{6}$, mais ces travaux - au demeurant intéressants - ne livrent qu'une vision incomplète du phénomène pour ne l'avoir envisagé qu'à une seule échelle - européenne ou régionale. Une telle mobilisation doit cependant être appréhendée de façon multiscalaire : à l'échelle européenne, pour rendre compte de l'importance globale du mouvement et de la façon dont la diplomatie pontificale l'a utilisé; à l'échelle nationale, puisque les conditions de la collecte ont dépendu de l'attitude des États; à l'échelle locale, afin d'examiner la mobilisation concrète des fidèles dans sa diversité; à l'échelle individuelle, enfin, pour rendre compte de la multiplicité des motifs qui ont pu pousser à aider la papauté.

Nous nous proposons ici d'aborder ces différentes dimensions à travers l'étude de la mobilisation financière des catholiques français, en nous appuyant sur des sources françaises, italiennes et vaticanes ${ }^{7}$. Les archives de la nonciature de Paris, conservées au sein des Archives secrètes vaticanes, sont à ce titre particulièrement précieuses. Elles contiennent notamment une importante correspondance des différents nonces à Paris concernant les dons et les souscriptions aux emprunts pontificaux. Surtout, y sont conservés les registres dans lesquels les représentants du Saint-Siège ont consigné mois après mois le montant des offrandes qu'ils recevaient, en précisant généralement le diocèse de provenance. Utilisé avec d'autres documents, et notamment les archives du ministère des Finances pontificales, conservées au

5. Carlo Crocella, Augusta miseria. Aspetti delle finanze pontificie nell'età del capitalismo, Milan, Nuovo istituto editoriale italiano, 1982. Sur les finances pontificales, les deux ouvrages de Benny Lai sont également utiles mais leur auteur ne cite pas ses sources : Benny Lai, Finanze e finanzieri vaticani tra l'Ottocento e il Novecento da Pio IX a Benedetto XV, Milan, Mondadori, 1979. Il faut aussi citer : Daniela Felisini, Le finanzie pontificie e $i$ Rothschild (1830-1870), Naples, Edizioni scientifiche italiane, 1991.

6. La meilleure étude locale est celle de Marius Faugeras, "Un aspect local de la question romaine : l'aide nantaise au Saint-Siège (1860-1870)", Annales de Bretagne et des pays de l'Ouest, 1983, tome 90, n 90-1, 1983, p. 47-72. Sur le rôle de la presse : Henri Sempéré, La Semaine catholique de Toulouse (1861-1908), Paris, Hachette, 1975. En ce qui concerne la France en général, on pourra se reporter avec utilité à la version non publiée de la thèse de Bruno Horaist : Envers Pie IX. La dévotion au pape et les catholiques français (1846-1878), thèse d'histoire sous la direction de Philippe Levillain, Université Paris X, 1993. Enfin, pour un exemple italien : Silvio Tramontin, «La raccolta dell'obolo di San Pietro a Venezia durant il pontificato di Pio IX (1860-1878) ", Pio IX, tome I, n 2, 1972, p. 295-309.

7. Le chercheur n'est pas qu'un pur esprit et est tributaire de certaines conditions matérielles; c'est pourquoi il nous faut remercier l'École française de Rome, qui, par les deux bourses qu'elle nous a octroyées, nous a permis de mener ces recherches. 
sein des Archives secrètes vaticanes et de l'Archivio di Stato de Rome, un tel registre permet d'avoir une connaissance relativement précise de l'importance que prit la mobilisation financière des catholiques français.

Il importe tout d'abord de retracer la genèse d'une telle mobilisation, en montrant la manière dont elle s'intégrait à la nouvelle stratégie d'appui sur les fidèles adoptée par la diplomatie pontificale au lendemain des événements de 1859-1860. L'étude de l'organisation du mouvement permet par ailleurs de mettre en évidence la diversité des soutiens dont la papauté pouvait bénéficier en France mais aussi les difficultés auxquelles elle dut faire face tout au long des années 1860. Enfin, l'analyse du montant et de la provenance des sommes recueillies doit aider à mieux saisir l'importance d'une mobilisation dont les significations furent multiples.

\section{UNE MOBILISATION SPONTANÉE DES FIDÈLES RAPIDEMENT ENCADRÉE PAR LA PAPAUTÉ}

Durant toute la première partie du XIX ${ }^{e}$ siècle, l'État pontifical eut à affronter de graves problèmes financiers, que la révolution romaine de 18481849 aggrava encore. À la fin des années 1850, pourtant, la situation s'améliorait. À la suite de plusieurs réformes qui augmentèrent substantiellement les revenus de l'État, le budget prévisionnel de 1859 atteignit même l'équilibre ${ }^{8}$. Les événements des années 1859-1860 mirent cependant fin à ces améliorations. La perte des Romagnes, qui s'étaient insurgées en 1859 à la faveur du conflit qui opposait dans le Nord de la péninsule italienne Français et Piémontais aux Autrichiens, puis celle des Marches et de l'Ombrie, annexées par le Piémont à la suite de sa victoire sur les troupes pontificales à Castelfidardo en 1860, privèrent le pape de ses provinces les plus peuplées et les plus riches, et par conséquent d'une part importante de ses revenus.

Alors que ceux-ci s'élevaient en 1858 à 12639000 écus romains ${ }^{9}$, ils n'étaient plus que de 5336000 en 1861, ce qui représentait une baisse de $58 \%$, alors que, dans le même temps, les dépenses ne baissèrent que de $33 \%$, pour atteindre 9764000 écus $^{10}$. Une part importante de celles-ci était en effet liée non à l'administration de l'État mais à celle de l'Église et, par ailleurs, le pape avait choisi d'honorer la totalité de la dette de ses États, considérant que le fait d'en laisser une partie à la charge du nouveau royaume d'Italie aurait équivalu à une reconnaissance de fait de la perte de ses provinces. Les finances pontificales eurent donc à affronter un déficit important pendant toutes les années $1860^{11}$. Cette situation, qui aurait dû provo-

8. Carlo Crocella, Augusta miseria..., op. cit., p. 35.

9. 18600 écus romains équivalent à 100000 francs, soit environ 1 écu pour 5,38 francs.

10. Carlo Crocella, Augusta miseria..., op. cit., p. 37-38.

11. Dans un article intitulé "Situation financière et politique du Saint-Siège", paru dans le 
quer une banqueroute, put cependant être maîtrisée grâce à l'aide financière apportée par des fidèles du monde entier.

Une telle mobilisation des catholiques en faveur des finances pontificales n'était alors pas nouvelle. En 1848-1849, déjà, lors de la précédente crise du pouvoir temporel des papes, Montalembert avait proposé de rétablir le denier de Saint-Pierre ${ }^{12}$ pour venir en aide à Pie IX et, dans plusieurs diocèses de France, l'œuvre avait de fait été établie et de l'argent collecté. Le retour du pape sur son trône avait cependant mis un terme à ces initiatives.

Ce ne fut donc qu'à la fin de l'année 1859 que se produisit le rétablissement durable du denier de Saint-Pierre. Dans plusieurs pays européens, des catholiques commencèrent alors à déposer, de manière spontanée, des offrandes pour le pape. Né en Grande-Bretagne, le mouvement s'étendit rapidement, et sans qu'aucune concertation ne se fît, à la Belgique, à l'Autriche et à la France ${ }^{13}$. Le caractère spontané des dons, dont l'initiative paraît avoir été due à des laïcs, témoignait bien de l'attention qui était alors portée à la question romaine dans toute l'Europe, pour des raisons à la fois religieuses et politiques.

Du point de vue religieux, tout d'abord, un tel intérêt s'expliquait largement par le processus de «mouvement vers Rome» (Mrr Salinis) qui caractérisait le catholicisme du XIX ${ }^{\mathrm{e}}$ siècle et avait considérablement renforcé la place du pape dans l'Église ${ }^{14}$. Les différentes crises du pouvoir temporel contribuèrent alors à renforcer la dévotion à Pie $\mathrm{IX}^{15}$, représenté dans une très abondante littérature catholique comme un pape "martyr" que les révolutionnaires cherchaient à dépouiller de son pouvoir temporel afin de le priver de son indépendance spirituelle.

À ces considérations religieuses s'ajoutaient des considérations politiques. Les révolutions de 1848-1849 ont en effet largement contribué à renforcer dans l'esprit des conservateurs européens le lien entre l'Église et le camp de l'ordre. En France, une telle situation est bien connue et illustrée par le basculement d'une partie de la bourgeoisie libérale conservatrice dans le cléricalisme au lendemain des Journées de Juin - Adolphe Thiers en est sans doute le meilleur exemple. Mais, plus largement, dans toute l'Europe, l'intérêt accordé à la question romaine change alors de nature. Celle-ci n'était jusque-là que l'une des composantes de la question italienne et n'intéressait les autres États européens qu'en ce qu'elle renvoyait aux rapports de force

Correspondant du 25 décembre 1869, Francisque de Corcelle le chiffre pour la période 1859-1868 à 289210000 francs, soit près de 30 millions par an.

12. Initialement, le terme désignait le tribut annuel remis au pape par le royaume d'Angleterre, du XIe siècle à 1534, en signe de vassalité. Cf. François-Charles Uginet, «Finances pontificales (époque contemporaine)" in Philippe Levillain [dir.], Dictionnaire historique de la papauté, Fayard, 1994, p. 688.

13. Carlo Crocella, Augusta miseria..., op. cit., p. 99.

14. Sur le mouvement vers Rome, $c f$. Philippe Boutry, «Le mouvement vers Rome et le renouveau missionnaire", in Jacques Le Goff et René Rémond [dir.], Histoire de la France religieuse, tome 3 sous la direction de Philippe Joutard, Paris, Le Seuil, 1991, p. 423-452.

15. Bruno Horaist, Envers Pie IX..., op. cit. 
entre les puissances ${ }^{16}$. La révolution romaine, sans faire disparaître cette dimension, en ajoute cependant une autre : pour les révolutionnaires comme pour les conservateurs, Rome apparait désormais comme le symbole de leur affrontement à l'échelle européenne et la question romaine se retrouve dès lors chargée d'une dimension politique particulièrement forte, qui explique que la papauté ait pu compter sur des soutiens dépassant très largement les seuls catholiques - Thiers en est, là encore, le meilleur exemple.

Considérations religieuses et considérations politiques s'entrecroisaient donc pour expliquer que dès la fin de l'année 1859, alors qu'il devenait clair que, malgré les stipulations de l'armistice de Villafranca et du traité de Zurich ${ }^{17}$, les Romagnes ne seraient pas rendues au pape, une mobilisation financière spontanée en faveur de la papauté ait eu lieu dans divers pays européens.

Rapidement cependant, la papauté chercha à encourager et à encadrer la mobilisation. Celle-ci offrait en effet une réponse à deux des principaux problèmes auxquels était alors confronté l'État pontifical. D'un point de vue financier, elle laissait espérer d'importantes rentrées d'argent, susceptibles de compenser partiellement le déficit de l'État romain. D'un point de vue politique, par ailleurs, elle s'accordait avec la volonté manifestée par une partie de la Curie de trouver dans le soutien des masses catholiques une compensation à la perte d'influence de la papauté auprès des souverains et gouvernements des puissances catholiques ${ }^{18}$. Dans le domaine militaire, une telle volonté, incarnée par Mrr de Mérode, pro-ministre des Armes de 1860 à $1865^{19}$, se manifesta par la création du corps des zouaves pontificaux, formé de volontaires venus du monde entier et censé pallier la défaillance de la protection de la France. Si le secrétaire d'État Antonelli ${ }^{20}$ ne cacha pas son opposition au projet de Mérode, dont il voyait bien le caractère illusoire, cela ne signifiait pas pour autant qu'il déniait tout intérêt à une stratégie d'appui sur les masses catholiques. Sa correspondance avec les différents nonces en France témoigne ainsi de l'importance qu'il accordait aux moyens d'influencer l'opinion publique, de manière à contraindre Napoléon III à adopter une politique plus favorable à l'État pontifical. La mobilisation financière des catholiques entrait pleinement dans ces vues, puisqu'elle fut considérée par

16. C'est d'ailleurs cette raison qui avait été la principale justification de l'expédition de Rome en 1849, qui visait ainsi à contrebalancer l'influence autrichienne en Italie. Maurice Agulhon, 1848 ou l'apprentissage de la République (1848-1852), Paris, Seuil, 2002 [1973], p. 106.

17. Les préliminaires de paix de Villafranca datent du 31 juillet, la paix de Zurich du 10 novembre. Ils avaient prévu la restauration des souverains italiens que des révoltes avaient chassés de leurs États ainsi que le retour des Romagnes sous la domination du pape.

18. Sur la stratégie diplomatique d'appui sur les masses catholiques, $c f$. Vincent Viaene, "The Roman Question. Catholic Mobilisation and Papal Diplomacy during the Pontificate of Pius IX (1846-1878)" in Emiel Lamberts (ed.), The Black International. L'Internationale blanche (1870-1878), Bruxelles/Rome, Institut historique belge de Rome, 2002, p. 135-177.

19. Roger Aubert, "Monseigneur de Mérode, ministre de la guerre sous Pie IX", Revue générale belge, mai-juin 1956, p. $1105-1116$ et 1316-1332.

20. Carlo Falconi, Il Cardinale Antonelli. Vita e carriera del Richelieu italiano nella chiesa di Pio IX, Milan, Mondadori, 1983. 
ses adversaires comme par ses partisans comme une manifestation politique en faveur du pouvoir temporel.

\section{Organisation et STRUCTURATION DE LA COLLECTE}

En février 1860, Antonelli invita le nonce à Paris à encourager les dons des fidèles ${ }^{21}$, qui commençaient alors à prendre une certaine importance ${ }^{22}$. La mobilisation s'était jusque-là développée localement, le plus souvent à l'échelle des diocèses, sans qu'aucune coordination nationale n'existât. Plusieurs évêques avaient ainsi, à partir de la fin de l'année 1859, incité dans leurs mandements et lettres pastorales les catholiques à déposer des offrandes pour le pape. À Paris, des dames de la bourgeoisie et de la noblesse s'étaient chargées de collecter des fonds ${ }^{23}$, qu'elles déposaient régulièrement à la nonciature $^{24}$.

Les articles organiques de 1802 limitaient cependant la possibilité pour les évêques de se concerter pour organiser la collecte à l'échelle nationale, si bien que seuls quelques groupes composés principalement de laïcs purent recueillir des fonds sur une vaste échelle. À Paris, un comité du denier de Saint-Pierre fut formé dans ce but. Ses membres appartenaient aux classes les plus élevées de la société et plusieurs d'entre eux, députés au Corps législatif, s'y illustrèrent dans la défense des intérêts du pape. Dès janvier 1860, le comité put remettre au nonce 200000 francs, dont une moitié était destinée au pape et l'autre au pro-ministre des Armes ${ }^{25}$. À Lyon, ce fut autour de Prosper Dugas que s'organisa à la même époque l'Euvre du denier de Saint-Pierre, bientôt rebaptisée Archiconfrérie de Saint-Pierre-ès-Liens après que Pie IX lui eut donné son approbation ${ }^{26}$. Elle se chargea de faire circu-

21. A. S. V. (Archives Secrètes Vaticanes), Nonciature de Paris, 185, Lettre d'Antonelli au nonce Sacconi du 4 février 1860.

22. A. S. V., Nonciature de Paris, 185, Lettre du nonce Sacconi à Antonelli du 21 février 1860. Sacconi dit avoir déjà reçu environ 60000 francs.

23. Si les femmes sont absentes des débats qui ont lieu à travers les journaux et brochures au sujet de l'unification italienne, elles occupent un rôle central dans la collecte du denier, dans le sillage du rôle qu'elles jouaient déjà auparavant dans les associations charitables. Il s'agit pour elles d'un des principaux vecteurs de politisation, permettant l'existence d'une "citoyenneté avant le vote», pour reprendre une expression de Magali della Sudda, Une activité politique féminine conservatrice avant le droit de suffrage en France et en Italie. Sociohistoire de la politisation des femmes catholiques au sein de la Ligue patriotique des Françaises (1902-1933) et de l'Unione fra le donne cattoliche d'Italia (1909-1919), thèse de doctorat d'histoire, sous la direction de Laura Lee Downs (EHESS) et Lucetta Scaraffia (Rome, Université La Sapienza), 2007.

24. A. S. V., Nonciature de Paris, 185, Lettre du nonce Sacconi à Antonelli du 21 février 1860.

25. On distingue les dons faits au titre du denier de Saint-Pierre, destinés théoriquement au pape mais en réalité transmis au ministre des Finances pontificales, M ${ }^{\text {gr }}$ Ferrari, et ceux destinés à l'armée pontificale, qui étaient remis au pro-ministre des Armes. Dans la pratique, bien souvent, l'appellation "denier de Saint-Pierre» englobe cependant les deux types de dons.

26. Sur Prosper Dugas, cf. Yves-Marie Hilaire et Jean-Marie Mayeur [dir.], Dictionnaire du monde religieux dans la France contemporaine. 6, Lyon. Le Lyonnais-Le Beaujolais, Paris, Beauchesne, 1994, p. 163-164. L'action de Dugas en faveur de la papauté s'inscrit plus généralement dans une série d'engagements en faveur du catholicisme ou de la cause légitimiste. Dans les années 1840, au moment 
ler des adresses destinées à témoigner au pape de l'intérêt que portaient les catholiques à sa cause, de collecter de l'argent et de diffuser des écrits populaires pour promouvoir la cause du pape dans les couches les moins favorisées de la sociétée ${ }^{7}$.

Les canaux par lesquels transitaient les dons étaient multiples et cette diversité perdura pendant toutes les années 1860 . Cela s'expliquait notamment par l'intérêt pour la papauté de s'appuyer à la fois sur les clercs et sur les laïcs. Les ecclésiastiques de rangs divers formaient un maillage du territoire français à la fois dense et hiérarchisé, ce qui offrait un cadre adapté aussi bien pour la collecte au niveau local que pour la transmission des fonds à Rome. Ainsi, une grande partie des dons, recueillis localement par un prêtre ou par un vicaire, étaient centralisés au niveau de l'évêché avant d'être transmis au nonce, qui lui-même déposait l'argent dans une banque afin que le ministère des Finances romain pût l'utiliser. Un tel circuit n'était cependant pas toujours suivi. Certains évêques - c'est le cas de l'évêque de Nantes ${ }^{28}$ - pouvaient en effet envoyer l'argent collecté au Comité de Saint-Pierre de Paris, qui lui-même le transmettait ensuite au nonce ${ }^{29}$. Un certain nombre de dons étaient par ailleurs directement transmis à Rome sans passer par la nonciature. Une note datant de 1867 et conservée au sein des archives du ministère des Finances pontificales témoigne à cet égard des difficultés que pouvait causer la multiplicité des canaux par lesquels transitait l'argent recueilli et d'une volonté d'homogénéiser les moyens de la collecte dans tous les pays en plaçant les laïcs sous la direction des prêtres, des évêques et des nonces ${ }^{30}$. La recommandation ne fut cependant pas suivie. Ainsi, lorsqu'en 1867 une tentative d'invasion garibaldienne vint rappeler aux catholiques la menace qui pesait toujours sur le pouvoir temporel du pape, une grande partie de l'argent collecté en France pour venir en aide à l'armée pontificale le fut non par les ecclésiastiques mais par différents journaux, nationaux ou locaux, qui organisèrent des souscriptions. Une telle situation témoignait du rôle acquis par les laïcs dans la défense des intérêts de l'Église depuis le début du XIX ${ }^{\mathrm{e}}$ siècle $^{31}$.

L'organisation de la collecte fut par ailleurs d'autant plus efficace que, dans bien des cas, elle s’appuyait sur des méthodes déjà employées en faveur d'autres œuvres religieuses. En témoigne par exemple la création à Lyon en 1866 de l'Euvre du Denier de saint Pierre (la première organisation de ce nom avait entre-temps disparu), constituée sur le modèle de l'Euvre de la

de la lutte pour la liberté d'enseignement, il crée ainsi la Gazette de Lyon, qui devient rapidement un journal légitimiste, et, en 1874, il soutient les carlistes espagnols.

27. Prosper Dugas, Vie et souvenirs, Poitiers, Oudin, 1878, p. 89-90.

28. Marius Faugeras, «Un aspect local...», loc. cit., p. 49-50.

29. A. S. V., Nonciature de Paris, 185, Lettre du nonce Sacconi à Antonelli du 16 juin 1860.

30. A. S. V., Secrétairerie d'État, $1870,110$.

31. Sur le nouveau rôle acquis par les laïcs dans la défense des intérêts de l'Église au milieu du XIX ${ }^{e}$ siècle, on nous permettra de renvoyer à Arthur Hérisson, "Louis Veuillot, L'Univers et l'intervention des laïcs dans les affaires de l'Église de France au milieu du XIXe siècle», Revue d'histoire de l'Église de France, tome 100, n² 245, juillet-décembre 2014, p. 333-354. 
Propagation de la Foi. Elle reprenait en effet à cette dernière le principe de la souscription régulière des fidèles (les feuilles de souscription prévoyant un montant d'un franc par an, ce qui permettait aux plus pauvres de participer), celui d'une organisation hiérarchisée en dizaines et centaines ${ }^{32}$ ainsi que celui de la publication d'un bulletin destiné à rendre compte des résultats tout en assurant le lien entre les associés ${ }^{33}$.

À quatre reprises - en $1860^{34}, 1863^{35}, 1864^{36}$ et $1866^{37}$-, l'État pontifical choisit par ailleurs, pour combler le déficit de ses finances, d'émettre de vastes emprunts dont la souscription était ouverte au public. Un tel procédé laissait en effet espérer un financement de la dette pontificale à moindre coût, en comptant sur le fait que la sympathie des souscripteurs à l'égard de la cause du pape les conduirait à accepter des taux moindres que ceux habituellement proposés ${ }^{38}$. En raison de la complexité de telles opérations, cellesci furent confiées à de grands banquiers catholiques. Dans le placement des emprunts, les ecclésiastiques se trouvaient dès lors dans une position d'auxiliaires, principalement chargés d'aider les représentants locaux des banques et d'encourager les fidèles à souscrire aux emprunts, ce qui n'allait pas sans poser des difficultés.

De telles difficultés n'étaient pas les seules rencontrées. Le consentement du gouvernement à une telle mobilisation financière était en effet fondamental alors même que, au début des années 1860, l'opposition catholique à sa politique italienne tendait à renforcer en son sein les tenants d'une position de fermeté à l'égard de l'Église ${ }^{39}$. Aussi, lorsque la collecte du denier de SaintPierre commença à prendre de l'ampleur, le ministre des Cultes Gustave Rouland déclara-t-il dans une circulaire datée du 5 mai 1860 que les comités formés afin de recueillir le denier de Saint-Pierre devaient se dissoudre, sous

32. De manière à faciliter la collecte, un chef de dizaine encadrait un groupe de dix souscripteurs et un centenier encadrait dix chefs de dizaine.

33. Bulletin de l'Euvre du denier de Saint-Pierre, organisée par souscriptions de 1 franc recueillies par dizaine, $\mathrm{n}^{\circ} 1$, Lyon, imprimerie Louis Perrin, 1866.

34. Il s'agissait d'un emprunt de 50 millions de francs au pair, dont le gouvernement pontifical avait obtenu du gouvernement français de pouvoir en placer 25 en France. Seuls 37 millions furent cependant souscrits par les catholiques, dont 17 en France (A. S. V., Nonciature de Paris, 138).

35. Il s’agissait de placer le solde du précédent emprunt, soit 13 millions, dont 8 pour la France, au taux de $74,5 \%$. En renonçant à placer son emprunt au pair, le gouvernement pontifical prenait acte des limites de la générosité des fidèles et de la nécessité de mieux rémunérer les souscriptions pour les rendre attrayantes (A. S. V., Nonciature de Paris, 138).

36. Il s'agissait d'un emprunt de 50 millions au pair. Seuls 25 millions furent souscrits (A. S. V., Nonciature de Paris, 190). Sur l'échec de cet emprunt, $c f$. Georges Jacquemyns, «L'attitude des évêques et des fidèles devant l'emprunt pontifical de 1864 ", Rassegna storica del Risorgimento, tome 50, $\mathrm{n}^{\circ} 1$, janvier-mars 1963 , p. 9-32.

37. Il s'agissait d'un emprunt de 60 millions, émis à 66 \% (A. S. V., Nonciature de Paris, 190). Seuls 38 millions furent souscrits (Carlo Crocella, Augusta miseria..., op. cit., p. 66).

38. A. S. V., Nonciature de Paris, 140, Note remise à S. Ex. M ${ }^{\mathrm{gr}}$ le Nonce Apostolique le 11 mars 1860 (non signée).

39. Témoigne notamment de ce changement d'attitude vis-à-vis des catholiques la suppression de L'Univers. Cf. Jean Maurain, La politique ecclésiastique du Second Empire de 1852 à 1859, Paris, Alcan, p. 373-381. 
peine de poursuites. Les journaux publièrent par la suite un communiqué explicitant la position du gouvernement :

«L'offrande individuelle est et reste libre; et qui ne pourrait la faire parvenir directement le peut par l'intermédiaire de son évêque ou de son curé. Mais ce que le gouvernement n'autorise pas, [...] c'est l'organisation de comités, de décuries enfermant dans les liens de leur organisation une armée de contribuables et agitant les esprits par les ardeurs de leur propagande. $»^{40}$

Le gouvernement craignait en effet que ne fussent organisées sous un prétexte religieux des manifestations politiques de défiance à l'égard de sa politique italienne. À Lyon, l'archiconfrérie de Saint-Pierre-ès-Liens fut ainsi dissoute en juillet $1860^{41}$.

Gustave Rouland permit simplement que fussent placés dans les églises des troncs $a d$ hoc où les fidèles pouvaient venir déposer spontanément des offrandes ${ }^{42}$; en réalité, des collectes purent être réalisées dans les églises, souvent à l'occasion des grandes fêtes chrétiennes. Le gouvernement se montra davantage bienveillant au sujet des emprunts pontificaux, alors même que la voie d'émission choisie - celle de souscriptions publiques - était inédite en France pour un emprunt émis par un gouvernement étranger ${ }^{43}$.

Si dans l'ensemble l'État, tout en cherchant à l'empêcher de prendre un caractère politique trop prononcée $e^{44}$, ne s'opposa pas à la mobilisation financière des catholiques français, celle-ci rencontra d'autres difficultés. La presse anticléricale laissa entendre que, loin d'aider les finances de l'État pontifical, les dons ne servaient qu'à enrichir encore davantage les principaux dignitaires de la Curie ${ }^{45}$. De même, au sujet des emprunts, des bruits furent répandus, selon lesquels les banques chargées de l'émission avaient offert à l'avance la somme mise sur le marché au pape et que, par conséquent, il était inutile de souscrire puisque l'argent reviendrait aux banques ${ }^{46}$.

40. Cité dans Idem, p. 430.

41. Archives nationales (Arch. nat.), $\mathrm{F}^{19} 1932$, Circulaire du ministre de l'Instruction publique et des Cultes aux évêques du 17 juillet 1860.

42. Arch. nat., $\mathrm{F}^{19}$ 1931, Lettre du ministre de l'Instruction publique et des Cultes à l'évêque d'Amiens du 17 novembre 1860.

43. A. S. V., Nonciature de Paris, 140, Lettre de Thouvenel au nonce Sacconi, 30 avril 1860. Une ordonnance de 1823 avait ouvert l'accès à la bourse de Paris aux emprunts étrangers, mais aucune disposition ne prévoyait qu'un emprunt émis par un gouvernement étranger pût se négocier par voie de souscription publique. Le ministre des Finances Magne proposa d'accorder ce droit à l'Etat pontifical sous certaines conditions.

44. On retrouve ici les mêmes craintes que celles exprimées pour justifier les mesures prises à la même époque contre la société de Saint-Vincent-de-Paul. Cf. Jean Maurain, La politique ecclésiastique..., op. cit., p. 554-567. Sur la société de Saint-Vincent-de-Paul : Mathieu Brejon de Lavergnée, La Société de Saint-Vincent-de-Paul au XIXe siècle. Un fleuron du catholicisme social, Paris, CERF, 2008.

45. C'est ce que signale notamment $\mathrm{M}^{\mathrm{gr}}$ de Ségur dans une petite brochure à 5 centimes destinée à une large diffusion (Le denier de Saint-Pierre, Paris, Tolra et Haton, 1861, p. 8-9).

46. A. S. V., Nonciature de Paris, 190, Prospectus du 5 juin 1866 sur les résultats de l'emprunt romain. 
Plusieurs évêques signalèrent par ailleurs les difficultés que rencontraient la collecte de dons et la souscription des titres d'emprunt. En octobre 1860, alors même que le nonce, pressé par Antonelli, venait d'écrire aux évêques de France pour leur demander d'encourager les dons dans leurs diocèses ${ }^{47}$, l'évêque de Verdun lui répondit ainsi qu'un appel à la générosité de ses diocésains lui semblait vain et qu'il avait jugé préférable de n'inviter que son seul clergé à participer ${ }^{48}$.

Malgré ces multiples difficultés, les collectes de fonds pour l'État pontifical se poursuivirent avec un certain succès pendant toute la décennie 1860 . Se pose dès lors la question des résultats concrets d'une telle mobilisation financière.

\section{LES RÉSULTATS DE LA MOBILISATION}

Chercher à évaluer les résultats de la mobilisation financière des catholiques en faveur de la papauté ne va pas sans poser problème. Dans un article intitulé "Situation politique et financière du Saint-Siège», paru dans Le Correspondant du 25 décembre 1869, Francisque de Corcelle donne les chiffres suivants :

\begin{tabular}{|c|c|}
\hline Année & Produit du denier de Saint-Pierre (en francs) \\
\hline 1861 & 14184000 \\
\hline 1862 & 9402000 \\
\hline 1863 & 7047000 \\
\hline 1864 & 5832000 \\
\hline 1865 & 6445000 \\
\hline 1866 & 5939000 \\
\hline 1867 & 11312000 \\
\hline 1868 & 11000000 \\
\hline
\end{tabular}

Ces chiffres permettent de distinguer trois phases principales : après des débuts particulièrement prometteurs, les dons diminuent pour se stabiliser entre 1863 et 1866 autour de six millions de francs ${ }^{49}$. À partir de 1867, cependant, une remontée peut être constatée, sans doute en raison à la fois des cérémonies du dix-huitième anniversaire du martyre de Pierre et de Paul, qui renforcent alors le prestige de la Rome pontificale, et du renouveau des menaces pesant sur le pouvoir temporel, illustré par la tentative d'invasion garibaldienne de $1867^{50}$. Parce que les archives des finances pontificales ne

47. A. S. V., Nonciature de Paris, 185, Lettre du nonce Sacconi aux évêques du 17 octobre 1860.

48. A. S. V., Nonciature de Paris, 185, Lettre de l'évêque de Verdun au nonce Sacconi.

49. La lassitude des fidèles peut expliquer cette période de faibles dons, de même que la signature en septembre 1864 d'une convention entre la France et l'Italie par laquelle ce dernier pays s'engageait à défendre l'intégrité de l'État romain et à prendre en charge une partie de sa dette, ce qui laissait espérer une résolution des problèmes financiers du Saint-Siège par la voie diplomatique.

50. L'analyse chronologique du montant des offrandes consignées dans les registres du nonce 
fournissent que des renseignements partiels, il est cependant difficile de s'assurer de la véracité des chiffres donnés par Corcelle.

Aux Archives d'État de Rome, Carlo Crocella avait trouvé un carton renfermant divers papiers concernant la collecte du denier de Saint-Pierre ${ }^{51}$. Si ceux-ci ne fournissent que des informations très lacunaires, ils offrent tout de même pour une année - 1864 - un tableau complet des dons reçus par la papauté. Ceux-ci s'élèvent à 1084 200,063 écus romains, ce qui correspond à peu de choses près à la somme donnée par Corcelle. Faut-il pour autant en conclure que les sommes que ce dernier donne pour les autres années sont justes? La prudence empêche de répondre à cette question avec certitude ${ }^{52}$.

L'évaluation des seuls dons provenant de France pose quant à elle d'autres problèmes. Parmi les papiers de la nonciature de Paris conservés au sein des Archives secrètes vaticanes se trouvent les registres dans lesquels les nonces consignèrent l'intégralité des dons au pape qui transitaient par eux. Ces différents registres couvrent l'ensemble des années 1860, avec une lacune pour les mois d'octobre et de novembre $1867^{53}$.

L'évaluation des dons français reste cependant difficile, en raison notamment de la diversité des circuits par lesquels transitaient les offrandes des fidèles. Le fait qu'une partie des dons recueillis localement n'arrivèrent jamais entre les mains du ministre des Finances à Rome ou même de celles du nonce mais furent utilisés localement par les comités qui les avaient collectés fait que ceuxci n'ont ainsi pas laissé de trace dans les comptabilités dont nous disposons ${ }^{54}$.

Par ailleurs, puisque l'intégralité des dons arrivés à Rome ne transitèrent pas par les nonces successifs, les registres de comptabilité établis par ceuxci ne suffisent pas pour établir la somme totale offerte par les catholiques français à Rome. Les 24258 084,03 francs passés par la nonciature entre le 15 février 1860 et le 31 décembre 1870 ne prennent en effet pas en compte les dons transmis directement au Saint-Siège. De plus, une telle somme inclut des offrandes recueillies hors de France mais envoyées à la nonciature de Paris. Les seules offrandes provenant de France et passées par la nonciature s'élèvent ainsi à environ 17,675 millions de francs.

Pour essayer de donner une estimation globale des dons provenant de France, il faut en réalité croiser les fonds des Archives secrètes vaticanes et ceux des Archives d'État de Rome. Ces derniers, on l'a dit, contiennent pour

laisse penser que cette hausse se poursuit jusqu'en 1869, avant une chute en 1870.

51. Archivio di Stato di Roma, Computista generale della R. C. A. Personale, affari generali, atti distribuiti per luoghi, 224.

52. Le même fonds de l'Archivio di Stato contient d'autres indications sur le montant total des dons reçus à Rome pour certaines années $(1863,1867,1868)$, montants qui correspondent dans l'ensemble, malgré quelques légers écarts, à ceux donnés par Corcelle.

53. A. S. V., Nonciature de Paris, 187 et 192 : les registres commencent le 16 octobre 1860 et finissent en 1872. En utilisant par ailleurs la correspondance contenue en A. S. V., Nonciature de Paris, 185, on peut remonter jusqu'au 15 février 1860.

54. Ceux-ci, notamment, étaient amenés à financer le voyage jusqu'à Rome des volontaires pontificaux, acheter des armes pour l'armée romaine ou encore venir en aide à d'anciens zouaves tombés dans le besoin. 
l'année 1864 un tableau recensant l'ensemble des dons reçus par la papauté. Un tel tableau donne la provenance de ces dons et offre par conséquent la possibilité d'évaluer le montant global offert par la France cette année-là ainsi que la part du pays dans le total des dons reçus par le Saint-Siège. Il permet dès lors de constater la place dominante des catholiques de France, qui envoient 2,5 millions de francs ${ }^{55}$, ce qui représente $43 \%$ du montant total des dons ${ }^{56}$. Parmi ces 2,5 millions de francs, un peu plus de la moitié $(53 \%)$ avait transité par la nonciature. En prenant pour hypothèse qu'un tel pourcentage a été relativement stable tout au long de la décennie 1860 - ce que rien ne nous permet d'affirmer avec certitude -, cela signifierait que le montant total des dons des catholiques français durant cette période peut être évalué à plus de 33 millions de francs. Un tel chiffre est considérable et témoigne de l'importance jouée par les catholiques français pour permettre la viabilité financière de l'État pontifical ${ }^{57}$.

Par ailleurs, puisque, les indications du nonce permettent de connaître le diocèse d'origine de $85 \%$ du montant total des dons provenant de France, il est possible de brosser une géographie des offrandes. Les deux cartes ci-contre représentent une tentative de cartographie de la mobilisation financière des catholiques français. Elles ont été élaborées en établissant pour chaque diocèse la somme des dons envoyés telle qu'on peut la retrouver dans les registres de la nonciature pour la décennie 1860-1870 [carte 1] et dans les registres de la nonciature et du ministère des Finances pour l'année 1864 [carte 2] ${ }^{58}$ en

55. Pour obtenir un tel chiffre, nous sommes parti du tableau fait par le ministère des Finances pontificales, n'avons conservé que les dons provenant de France et supprimé les lignes «nonciature de Paris", étant donné que transitaient par celle-ci des dons provenant d'autres pays. Ensuite, il nous a suffi d'ajouter les dons signalés comme provenant de France dans le registre de la nonciature de Paris pour 1864.

56. La France est ainsi de très loin le pays qui contribue le plus au denier de Saint-Pierre. La contribution belge, rapportée à la population du pays, est toutefois supérieure à la française.

57. Cette estimation est sans doute une estimation basse car elle ne prend pas en compte l'ensemble des dons, puisque d'une part certains d'entre eux ne parvinrent jamais à Rome mais furent employés sur leurs lieux de collecte et que d'autre part la nonciature ne semble pas avoir inclus dans ses registres les sommes qu'elle recevait pour l'armée pontificale avant mars 1868, sinon de manière occasionnelle. À titre de comparaison, au cours des années 1860, les revenus de l'Euvre pour la Propagation de la Foi - qui ne paraît pas avoir pâti de la concurrence du denier de Saint-Pierre - furent d'environ trois millions de francs par an en France (Michel Lagrée, "Les versements des diocèses français à l'Euvre de la Propagation de la Foi» in Marcel Paco et Olivier Fatio, L'Hostie et le denier. Les finances ecclésiastiques du haut Moyen Age à l'époque moderne, Genève, Labor et Fides, 1991, p. 287). En 1860, la société de Saint-Vincent-de-Paul recueillait quant à elle 2,2 millions de francs dans le pays (Mathieu Brejon de Lavergnée, La Société..., op. cit., p. 274). Les trois ou quatre millions recueillis chaque année au titre du denier de Saint-Pierre constituent donc une somme importante, supérieure à celles réunies par les autres grandes œuvres catholiques. Le caractère exceptionnel de la collecte tient cependant davantage encore en la rapidité de son organisation : alors que l'Euvre pour la Propagation de la Foi n'avait atteint les trois millions de francs récoltés annuellement que près de quarante ans après sa fondation, le denier de Saint-Pierre dépassait cette somme dès sa première année.

58. L'utilisation conjointe de ces deux cartes est rendue nécessaire par le fait que chacune d'entre elles a le mérite de combler partiellement les lacunes de l'autre : la carte 1 permet une vision sur la longue durée, tandis que la carte 2 rend leur importance aux diocèses qui envoyaient leurs offrandes directement à Rome sans passer par le nonce. 


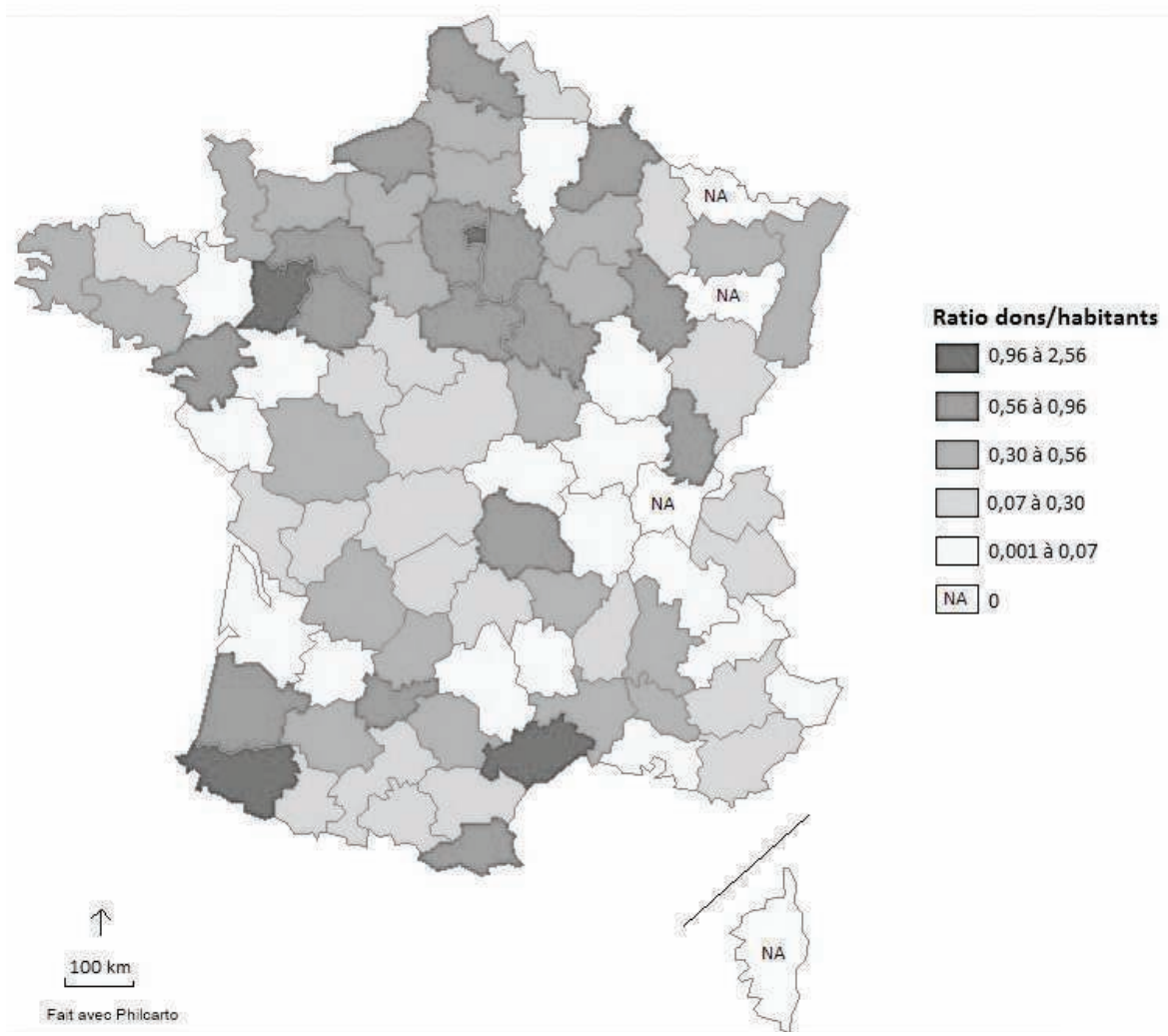

Carte 1. Provenance des dons au pape transmis à la nonciature de Paris de 1860 à 1870

rapportant ce montant des dons à la population ${ }^{59}$, de manière à supprimer les biais liés à l'inégal poids démographique des diocèses de France ${ }^{60}$.

Les enseignements qui peuvent être tirés de ces cartes sont multiples. La sous-représentation de certaines régions, et notamment de celles du Midi, dans la carte 1 montre l'existence de plusieurs réseaux par lesquels transitaient les dons au pape. À ce titre, il n'est pas illogique que les diocèses les

59. Les chiffres utilisés sont ceux de la Statistique de la France pour l'année 1866 : Statistique de la France, deuxième série, tome XVII, Résultats généraux du dénombrement de 1866, Strasbourg, Imprimerie administrative de Veuve Berger-Levrault, 1869.

60. Au XIX ${ }^{\mathrm{e}}$ siècle, les diocèses correspondent généralement aux départements. Il existe cependant quelques exceptions, dont nous avons tenu compte. Lorsqu'un même département englobait deux diocèses, nous avons fusionné les dons des diocèses en question pour les diviser par la population du département. Lorsqu'à l'inverse un diocèse englobait deux départements, nous avons divisé le total des dons du diocèse par la somme de la population des deux départements. Enfin, deux cas étaient plus complexes en raison de la non concordance des frontières diocésaines et départementales. Le diocèse de Reims correspondait ainsi au département des Ardennes auquel s'ajoutait l'arrondissement de Reims (le diocèse de Châlons correspondait par conséquent au département de la Marne moins l'arrondissement de Reims) et le diocèse de Fréjus correspondait au département du Var auquel s'ajoutait l'arrondissement de Grasse (le diocèse de Nice correspondait par conséquent au département des Alpes-Maritimes moins l'arrondissement de Grasse). La Statistique de la France a permis de recalculer la population de chacun des diocèses concernés. 


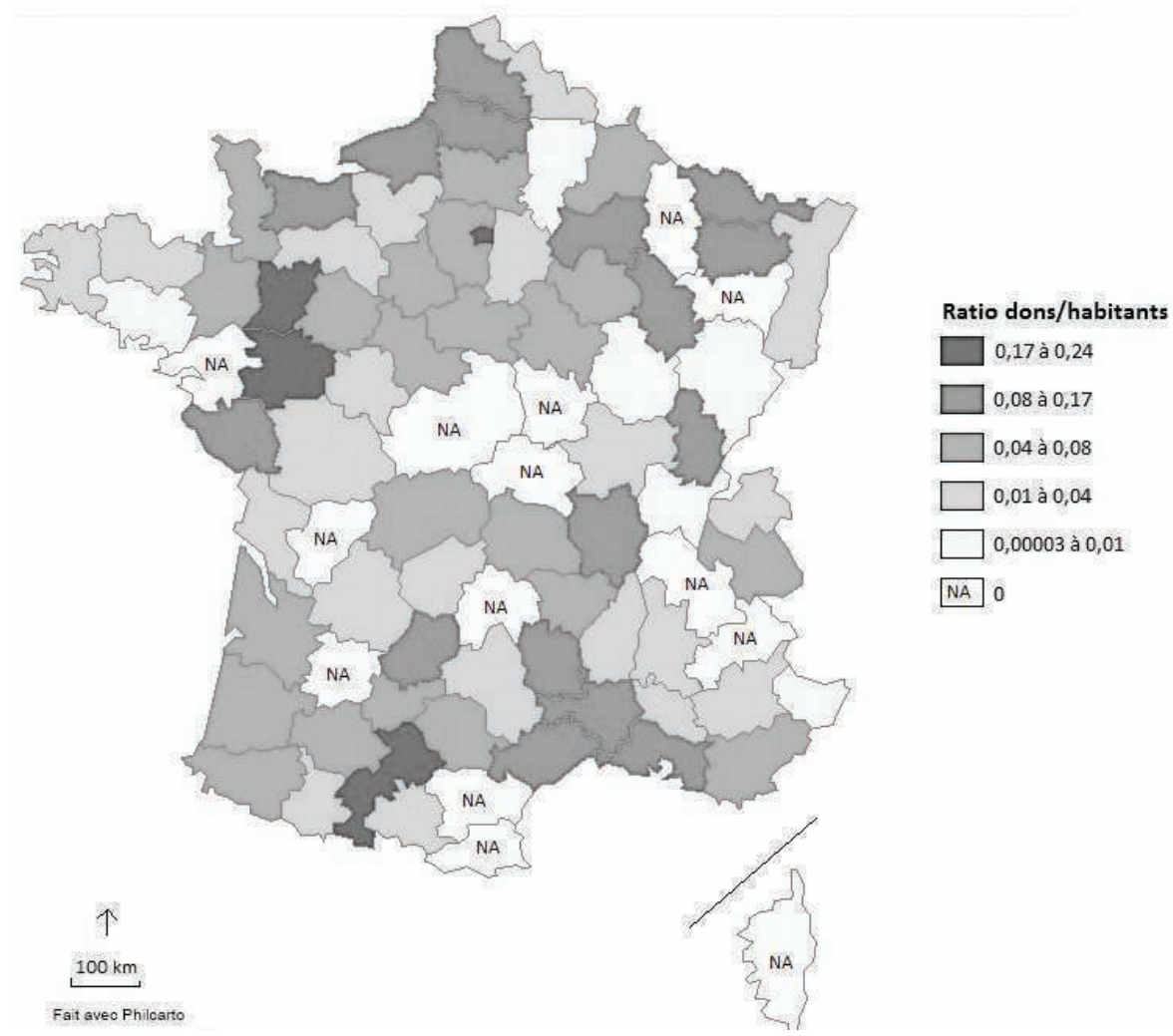

Carte 2. Provenance des dons au pape en 1864

plus proches de l'Italie aient directement envoyé leurs offrandes à Rome plutôt que de les faire passer par la nonciature. Il faut donc distinguer au moins deux réseaux de transmission des dons, le premier centré autour de Paris et le second autour de Marseille, où la présence d'un consul pontifical ainsi que l'existence d'une liaison maritime directe avec l'État pontifical ont dû favoriser une certaine centralisation des dons.

La géographie des offrandes au pape telle que l'on peut la saisir à travers ces deux cartes semble par ailleurs renvoyer à différentes réalités de la France du XIX siècle. On retrouve tout d'abord largement les régions les plus ferventes, qu'il s'agisse du Midi, où Montpellier et Nîmes jouent notamment un rôle central, du Pays basque, du grand Ouest, où le poids de Nantes ${ }^{61}$, d'Angers et de Laval est particulièrement important, du Nord et de l'Est ${ }^{62}$.

61. Le diocèse de Nantes n'apparaît pourtant qu'à une seule reprise dans les registres avant janvier 1866 parce que les dons qui y étaient recueillis n'étaient pas transmis au nonce : l'importance de sa contribution aux finances pontificales est donc largement sous-évaluée sur les cartes. De fait, alors que les registres du nonce ne signalent que 434368,50 francs de dons, Marius Faugeras évalue, à partir des archives diocésaines, les dons du diocèse à 1 million de francs.

62. On remarquera que la carte coïncide partiellement - mais seulement partiellement - avec la 
La ferveur religieuse ne suffit cependant pas à rendre compte de la géographie des dons. La richesse des territoires semble également avoir eu un rôle important, comme le montre la surreprésentation des diocèses situés au nord de la ligne Saint-Malo-Genève. Enfin, en lien d'ailleurs avec la question de la richesse, on trouve aussi surreprésentés les diocèses les plus urbains. Le diocèse de Paris est ainsi de loin celui qui envoie le plus d'argent au pape mais les villes de Lyon, Marseille, Rouen, Nantes ou encore Toulouse sont aussi à signaler. On peut supposer que, dans les villes, la collecte du denier a bénéficié de l'existence préalable de réseaux de collecte pour les œuvres de charité, qui ont été réutilisés dans les années 1860 au profit du pape.

Les donateurs ne se limitaient cependant pas aux seuls membres des classes aisées urbaines. Les listes de souscription publiées à diverses reprises dans les journaux laissent en effet voir la grande diversité sociale des personnes concernées. Ces listes précisent en effet généralement le nom des donateurs ${ }^{63}$ et parfois leur métier. On y constate ainsi que, si les classes aisées de la population sont dominantes, des membres des couches populaires participent cependant également. Ces listes permettent par ailleurs de noter l'importance de la présence des femmes dans le soutien à la cause pontificale. Dans celles que publient L'Univers du 15 au 23 octobre $1867^{64}$, au moment où une tentative d'invasion garibaldienne menace Rome, les femmes contribuent à hauteur d'environ $20 \%$ du montant total des dons. La défense du pape devient ainsi pour certaines d'entre elles un des espaces alternatifs qui leur permettent de participer à la vie de la Cité alors même qu'elles ne disposent pas du droit de vote. L'existence d'associations exclusivement féminines, comme l'Euvre des zouaves pontificaux formée à Montpellier en $1869^{65}$, témoigne par ailleurs de la relative autonomie dont elles pouvaient bénéficier.

\section{LA SIGNIFICATION DE LA MOBILISATION}

Reste à s'interroger sur la signification d'un tel mouvement. La dimension religieuse de ces dons dans la très grande majorité des cas ne fait guère de doute, même si le conformisme et la volonté de ne pas déplaire au prêtre ou au notable qui s'occupait de la collecte a pu sans doute motiver certaines offrandes. Que les sommes recueillies aient été aussi massives témoigne de l'ouverture européenne du catholicisme français au milieu du XIX ${ }^{\mathrm{e}}$ siècle. La piété ne se conçoit alors plus uniquement dans le simple cadre de la pratique

carte de la pratique religieuse de la France rurale établie au milieu du XX $\mathrm{XX}^{\mathrm{e}}$ siècle par Fernand Boulard et Gabriel le Bras, en mettant notamment en évidence le poids des périphéries catholiques de la France.

63. Un tel phénomène de publicité des noms des donateurs n'a rien d'original en matière de souscription politique. Cf. par exemple Emmanuel Fureix, La France des larmes. Deuils politiques à l'âge romantique (1814-1840), Seyssel, Champ Vallon, 2009, p. 367.

64. Pour ces neuf jours, les listes mentionnent 1614 dons pour un total de 69699,95 francs.

65. Annales du denier de Saint Pierre. Bulletin des auvres pontificales, IX, 1869, p. 285. 
locale et quotidienne du culte mais induit également un rapport, de plus en plus important à cette époque, à la papauté comme centre de la catholicité.

À cette signification religieuse s'ajoute par ailleurs dans bien des cas une dimension politique. Donner au denier de Saint-Pierre revenait pour beaucoup à afficher son soutien à l'existence de l'État pontifical. Une telle signification est encore plus nette dans les dons en faveur de l'armée pontificale ainsi que dans la souscription des emprunts romains qui, contrairement au denier de Saint-Pierre, qui était officiellement une offrande des fidèles à la personne du pape, était quant à elle ouvertement destinée à l'État pontifical. L'autre signification politique que pouvaient revêtir les dons était liée à la place symbolique occupée par Rome et la papauté dans le conflit entre révolution et contre-révolution, qui faisait de la cité pontificale un des lieux centraux de ce que les historiens ont pu qualifier, selon qu'ils prêtaient davantage leur attention aux questions politiques ou aux questions religieuses, d' «Internationale blanche ${ }^{66}$ ou d' "Internationale noire ${ }^{67}$. Donner à Pie IX, c'était donc dans bien des cas soutenir contre la révolution la cause de la légitimité, comme en témoigne la lettre envoyée au nonce par le comte Arthur de Beaumont, le 15 juin 1860, pour lui transmettre les dons qu'il avait recueillis dans la paroisse de Saint-Hilaire de Talmond (Vendée) :

"Nos cœurs de Royalistes et de chrétiens toujours prêts à soutenir la cause de la religion et de la légitimité, gémissent du succès de la révolution qui fait le tour du monde, ainsi que de la persécution qui a commencée contre notre sainte religion mais, quoique [sic] il arrive, nous serons toujours fidèles à notre Dieu et à notre Roi! ${ }^{68}$

Qu'il s'agisse de soutenir l'État pontifical ou de défendre à travers le pape la cause de la légitimité, le don prend alors une valeur de méfiance voire d'hostilité à l'égard du Second Empire, ce qui explique les craintes un temps manifestées par les autorités à l'égard des collectes. Reste cependant qu'une telle dimension politique n'est pas présente chez tous les donateurs : pour un catholique républicain comme Arnaud de l'Ariège, par exemple, le denier de Saint-Pierre avait au contraire pour vocation de rendre inutile le maintien

66. Sur la genèse et la structuration de réseaux de solidarité transnationale entre les contrerévolutionnaires européens, on pourra se reporter, en plus de la thèse de Simon Sarlin déjà citée, à Jordi Canal, "Guerres civiles en Europe au XIX ${ }^{e}$ siècle, guerre civile européenne et Internationale blanche", in Jean-Paul Zúñiga [dir.], Pratiques du transnational. Terrains, preuves, limites, Paris, Centre de Recherches Historiques, 2011, p. 57-77 et Jean-Clément Martin [dir.], La contre-Révolution en Europe. XVIII'-XIXe siècles. Réalités politiques et sociales, résonances culturelles et idéologiques, Rennes, Presses Universitaires de Rennes, 2001 ainsi qu’à Alexandre Dupont, Une Internationale blanche. Les légitimistes français au secours des carlistes (1868-1883), thèse de doctorat en histoire, Université Paris-1 et Universidad de Zaragoza, 2015, $980 \mathrm{f}^{\circ}$.

67. Emiel Lamberts (ed.), The Black International..., op. cit.

68. A. S. V., Nonciature de Paris, 185. 
du pouvoir temporel du pape en offrant à ce dernier une autre source de revenus ${ }^{69}$.

Enfin, on peut également retrouver derrière les dons des considérations d'une autre nature, qui renvoient aux luttes d'influence et de prestige au sein du champ catholique. Lorsque le don avait, d'une manière ou d'une autre, une forme de publicité, une compétition pouvait exister entre certains acteurs pour apparaitre comme étant celui qui s'était montré le plus généreux vis-à-vis du pape. En témoignent par exemple les listes de souscriptions pour l'armée pontificale publiées par divers journaux à la fin de l'année 1867, au moment où l'invasion garibaldienne des États pontificaux avait rendu d'autant plus nécessaire le renforcement des troupes du pape. L'Univers, publie ainsi des listes du 15 octobre 1867 au 16 avril 1868 pour un montant total de 901880,04 francs. Le 15 mars, il signale le montant des dons recueillis par les autres journaux parisiens, montant dont le total est inférieur à la somme qu'il a recueillie à lui seul. Par la publication de tels chiffres, les rédacteurs du journal cherchaient sans nul doute à renforcer leur position aussi bien par rapport à la papauté que par rapport aux catholiques français.

Grâce à la mobilisation financière des catholiques, l'État pontifical réussit pendant toutes les années 1860 à limiter la gravité des problèmes financiers auxquels il était confronté. Certes, les dons des fidèles étaient loin de suffire à combler le déficit chronique de ses finances, mais ils offraient de nouveaux revenus réguliers qui pouvaient servir de garantie aux emprunts de l'État.

De 1860 à 1870, les offrandes provenant de France s'élevèrent vraisemblablement à trente ou quarante millions de francs, somme considérable ${ }^{70}$ qui représentait environ une année de déficit de l'État romain. En plus de l'importance qu'elles avaient d'un point de vue économique, elles montraient l'intérêt des Français pour la question romaine et plus généralement pour les affaires italiennes. Au contraire du volontariat armé international, qui ne concerna jamais qu'un très petit nombre de personnes ${ }^{71}$, la mobilisation financière des catholiques témoigne ainsi du fait que la question italienne fut suivie avec attention par une grande partie des Français et qu'elle fut même l'occasion pour un nombre non négligeable d'entre eux d'engagements concrets, dont les dons au pape ne furent que l'une des nombreuses formes possibles.

Une telle mobilisation montrait par ailleurs que l'Église, en ce milieu de $\mathrm{XIX}^{\mathrm{e}}$ siècle, était capable d'entraîner dans sa défense une part importante de la population française, en s'appuyant non seulement sur les structures tra-

69. Frédéric Arnaud de l'Ariège, L'indépendance du Pape et les droits des peuples, Paris, Dentu, 1860, p. 6-7.

70. Les sommes recueillies chaque année sont par elles-mêmes importantes, mais ce qui distinguait surtout le denier de Saint-Pierre des autres souscriptions politiques qui avaient pu être lancées dans les décennies précédentes en France, c'était le caractère durable de ces dons.

71. On évalue à 3000 le nombre de Français partis s'engager au sein des zouaves pontificaux. 
ditionnelles de la paroisse et du diocèse, qui opéraient un maillage dense et hiérarchisé du territoire français, mais aussi sur des moyens plus modernes, comme la presse, que le rôle pris par les laïcs dans la défense des intérêts religieux lui permettait d'utiliser. En cela, la mobilisation autour de la question romaine apparaît comme une étape importante dans le processus de politisation des catholiques, placée à la suite de la longue lutte en faveur de la liberté d'enseignement des années $1840^{72}$ et annonçant les futurs combats de la Troisième République.

Arthur Hérisson est doctorant contractuel enseignant Centre d'Histoire du XIXe siècle (Universités Paris 1-Paris 4) dans la France des notables (1830-1850), Paris, Honoré Champion, 2015. 